soleus muscle. Twenty healthy volunteers with an average age of 29 years underwent an ultrasound of the right PTF joint. The joint line spacing was visualized in $100 \%$ of the cases on the 3 sections. The anterior cross-section allowed anterior proximal tibiofibular ligament analysis in $100 \%$ of the cases (median length $15,7 \mathrm{~mm}$ (min-max: $12.3-23.4$ ), median thickness $1.4 \mathrm{~mm}$ (min-max: 1-2.3). The coronal section allowed identification of the inferolateral geniculate and posterior tibial recurrent arteries in 90 and $85 \%$ of cases respectively, and the distal insertion of the fibular collateral ligament in $100 \%$ of cases. Posterior sectioning was more challenging and identification of the popliteal tendon, arcuate ligament and posterior proximal tibiofibular ligament was possible in 16, 7 and 2 patients respectively.

Conclusion: We performed a 2-step study: a cadaveric study followed by an ultrasound on healthy volunteer which allowed us to define 3 standardized scan of the PTF joint. These sections allow a thorough study of the PTF joint and the surrounding structures although study of the posterolateral corner ligaments remains challenging. We think that this scanning method can be integrated into daily clinical practice in rheumatology and in sports medicine.

Disclosure of Interests: None declared

DOI: 10.1136/annrheumdis-2020-eular.3929

\section{AB1136 CONNECTIVE TISSUE DISEASE ASSOCIATED INTERSTITIAL LUNG DISEASE IN THE UYGUR POPULATION OF CHINA.}

X. Wu ${ }^{1}$, L. Wu ${ }^{1}$, C. N. Luo ${ }^{1}$, Y. M. Shi' ${ }^{1}{ }^{1}$ People's Hospital of Xinjiang Uygur Autonomous Region, Urumqi, China

Background: Connective tissue diseases are a group of inflammatory, immune mediated disorders. Interstitial lung disease (ILD) is associated with significant morbidity and mortality.Currently,, scientists are still looking for serum markers to diagnose interstitial lung disease.Althought serum KL-6 level has been studied in ILD of various aetiologies and revealed to be an important serum marker for ILD, but differences in KL-6 expression related to ethnic and/or genetic variants may exist.

Objectives: To evaluate the diagnosis of the serum Krebs von den Lungen-6 (KL-6) for CTD-ILD in the Uygur population of China.

Methods: 117 Patients with CTD-ILD (CTD-ILD group) and 182 patients with CTD (CTD group) who visited the department of rheumatology and immunology of People's Hospital of Xinjiang Uygur Autonomous Region between January, 2015 and December, 2019 were included. Serum KL- 6 levels were measured by chemiluminescent enzyme immunoassay kit.

Results: The significantly higher levels of KL- 6 were determined in the RA-ILD group than RA group $[569(287.5,984) \mathrm{U} / \mathrm{ml}$ vs $194(152,266.5) \mathrm{U} / \mathrm{ml}](\mathrm{P}<0.001)$ (figure 1). The optimal cutoff value of serum KL-6 for diagnosis of RA-ILD was $345.5 \mathrm{U} / \mathrm{ml}$, and the sensitivity and specificity were $71.8 \%$ and $90.1 \%$, respectively. Area Under the Curve (AUC) was 0.875. (figure 2)

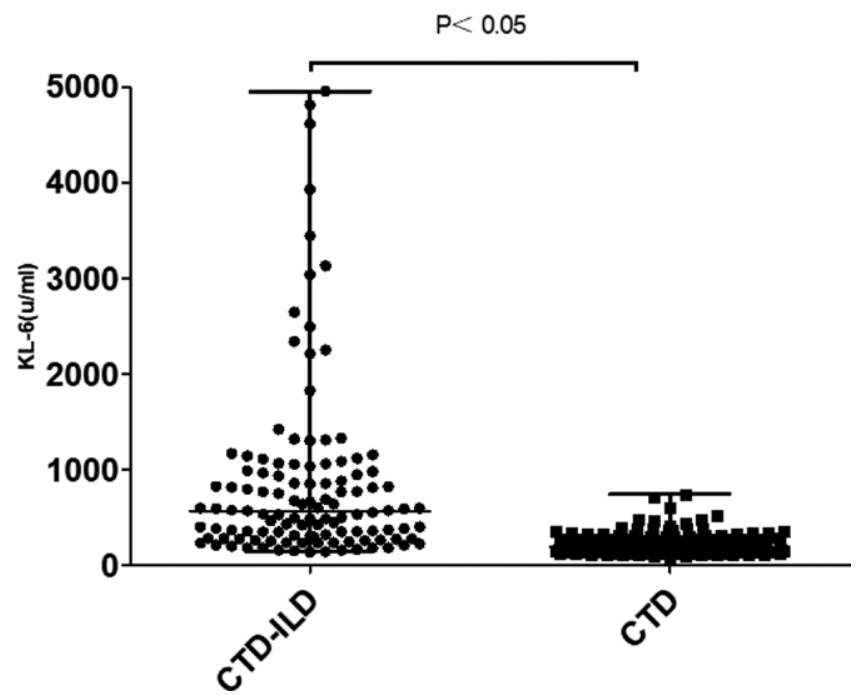

Figure 1. Comparison of serum KL-6 concentrations in CTD-ILD group and CTD group.

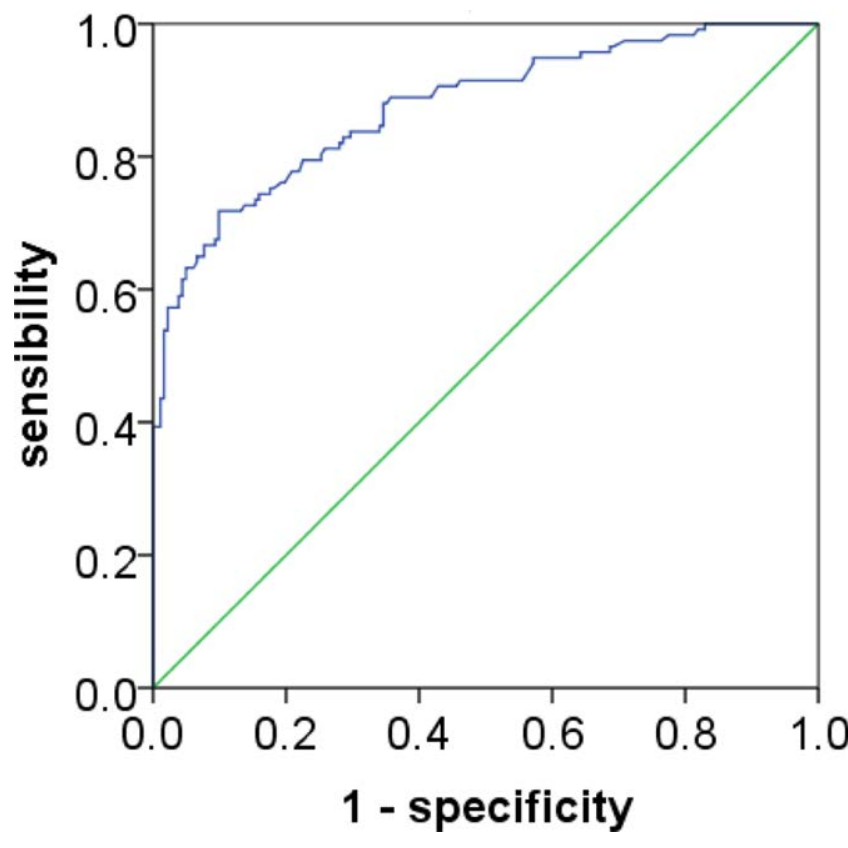

Fig 2. Receiver-operating characteristic curve(ROC) of KL-6 for the diagnosis of CTD-ILD

Conclusion: The serum KL-6 is a important biomarker for the diagnosis of CTD ILD and Serum KL-6 could be a clinically useful biomarker in screening CTD-ILD in the Uygur population of China.

References:

[1] Woodhead F, Wells A U, Desai S R. Pulmonary Complications of Connective Tissue Diseases[J]. Clinics in Chest Medicine, 2008, 29(29):149-164.

Tanaka S, Hattori N, Ishikawa N, et al. Krebs von den Lungen-6 (KL-6) is a progn -ostic biomarker in patients with surgically resected nonsmall cell lung cancer. Int J Cancer 2012; 130:377-87.

[2] Ogz E O, Kucuksahin O, Turgay M, et al. Association of serum KL-6 levels with interstitial lung disease in patients with connective tissue disease: a cross-sectional study. Clinical Rheumatology, 2016, 35(3):663-666.

Disclosure of Interests: None declared

DOI: 10.1136/annrheumdis-2020-eular.4860

\section{AB1137 1 CLASSIFICATION OF THE EARLY STAGE OF RAPIDLY DESTRUCTIVE COXOPATHY ACCORDING TO THE FEMORAL HEAD DESTRUCTION}

T. Yasuda ${ }^{1}$, K. Matsunaga ${ }^{1}$, T. Hashimura ${ }^{1}$, Y. Tsukamoto ${ }^{1}$, T. Sueyoshi ${ }^{1}$, S. Ota ${ }^{1}$, S. Fujita ${ }^{1}$, E. Onishi ${ }^{1}{ }^{1}$ Kobe City Medical Center General Hospital, Department of Orthopaedic Surgery, Kobe, Japan

Background: Rapidly destructive coxopathy (RDC) is an unusual subset of osteoarthritis of the hip characterized by rapid chondrolysis with progressive loss of the joint space as the first manifestation of the disease. Because rapid progression of RDC makes it difficult to obtain sequential radiographs in its early stage, the process of disease progression in the early stage remains unclear. Although the pathogenesis of RDC is still unclarified, the potential causes of RDC include subchondral insufficiency fracture of the femoral head resulting from osteoporosis, pelvic posterior inclination in RDC as a mechanical factor, and increased serum levels of matrix metalloproteinase (MMP)-3 as a biological factor.

Objectives: This study aimed to differentiate the process of disease progression in the early stage of RDC and provide its new classification system.

Methods: This monocentric retrospective study included 42 female patients who met the criteria of $\mathrm{RPOH}$, chondrolysis $>2 \mathrm{~mm}$ during 12 months from the onset of hip pain based on a series of radiographs and computed tomography (CT). This study also included 9 female patients with osteoarthritis secondary to developmental dysplasia of the hip (DDH), who demonstrated chondrolysis $>2 \mathrm{~mm}$ during 12 months from the onset of hip pain. Cortical thickness index (CTI) correlated with bone mineral density of the hip, pelvic tilt, and serum concentrations of matrix metalloproteinase (MMP)-3 were analyzed.

Results: RDC were classified into two types based on the absence (type 1, $\mathrm{n}=17$ ) and presence (type 2, $\mathrm{n}=25$ ) of subsequent femoral head destruction shown by CT within 12 months after the onset of hip pain. MMP-3 significantly 
increased in RDC type 2 compared with type 1 and DDH. Increased posterior pelvic tilt was found in RDC type 2 compared with $\mathrm{DDH}$. Logistic regression and receiver operating characteristic curve analyses indicated that MMP-3 may be associated with differentiation between RDC types 1 and 2. No difference was found in CTI between RDC types and DDH.

RDC type 2 hips developed partial (type 2A) and massive (type 2B) femoral head destruction within the first 12 months. Whereas partial destruction showed $<20 \%$ collapse ratio, massive destruction demonstrated $>40 \%$ collapse ratio. Increased posterior pelvic tilt was found in massive destruction. Femoral head destruction started earlier within the first 6 months in massive destruction compared with that in partial destruction. From receiver operating characteristic curve analysis, pelvic tilt differentiated the femoral head destruction types using the initial radiograph at the onset before first demonstration of femoral head destruction. No difference was found in $\mathrm{CTI}$ or MMP-3 between the two subtypes.

Conclusion: Disease progression of RDC during 12 months after the onset of hip pain could be classified into two distinct types based on the absence (type 1) and presence (type 2) of femoral head destruction in association with MMP-3 and pelvic tilt as biological and mechanical factors, respectively. MMP-3 may be helpful to differentiate those two types in the early stage of RPOH. The extent of femoral head destruction could further differentiate RDC type 2 into two subtypes based on pelvic tilt.

Acknowledgments : This study was supported by the Japan Hip Joint Foundation.

Disclosure of Interests: None declared

DOI: 10.1136/annrheumdis-2020-eular.347

\section{AB1138 ASSESSMENT OF FLUORESCENCE-OPTICAL IMAGING TECHNIQUE OF THE HANDS IN PSORIASIS AND PSORIATIC PATIENTS USING AN INNOVATIVE OBJECTIVE METHOD}

L. Zerweck ${ }^{1,2}$, U. Henkemeier ${ }^{2,3,4}$, P. H. Nguyen ${ }^{1,2}$, T. Rossmanith ${ }^{2,3}$, A. Pippow ${ }^{1,2}$, H. Burkhardt ${ }^{2,3,4,5}$, F. Behrens ${ }^{2,3,4,5}$, M. Köhm ${ }^{2,3,5}{ }^{1}$ Fraunhofer Insitute for Applied Information Technology, Sankt Augustin, Germany; ${ }^{2}$ Fraunhofer Cluster of Excellence Immune-Mediated Diseases, Frankfurt, Germany; ${ }^{3}$ Fraunhofer IME Translational Medicine and Pharmacology, Frankfurt, Germany; ${ }^{4}$ Centre of Innovative Diagnostics and Therapeutics Immunology/ Rheumatology CIRI, Frankfurt, Germany; ${ }^{5}$ Goethe-University Frankfurt, Frankfurt, Germany

Background: Psoriasis (Pso) is one of the most common chronic inflammatory skin diseases in Europe. Psoriatic arthritis (PsA) is closely associated to Pso whereas the skin manifestation appears usually years before PsA-related symptoms emerge. Up to $30 \%$ of Pso patients develop PsA, biomarkers for its early detection are of major importance. In early PsA, changes in synovial vascularisation appear first. Imaging biomarkers for detection of changes in vascularisation might be useful for early detection of musculoskeletal disease. Fluorescence-optical imaging (FOI) is a new method to detect changes in microvascularisation of the hands. Each collected data set of the FOI system contains 360 images representing a time progression of the indocyanine green (ICG) distribution.

Objectives: To evaluate a reader-independent assessment method for evaluation of FOI in patients with $\mathrm{PsO}$ and PsA.

Methods: A prospective study including patients with dermatological confirmed skin PsO was performed. 411 patients were included from German dermatology units without PsA diagnosis but potential risk for its development. Clinical examination (CE) was performed by a qualified rheumatologist. For a reader independent evaluation of the FOI images an objective joint-based scoring method was developed. For this method, the joint areas are defined by image segmentation and scored based on generated heatmaps. To calculate a heatmap indicating conspicuous joints from a data set containing 360 images, each pixel is converted to a time series containing 360 values. From this time series, three independent values (features) are extracted: amplitude, average value and maximal slope. Thus, each pixel is reduced to three different feature values. After the three features are determined for each pixel, k-means clustering is performed on each feature. The numbers of centroids (k) are set to 3,5 , 7 and 9.12 heatmaps ( 3 features à $4 \mathrm{ks}$ ) are calculated, which results in 12 scores for each joint as well. The clusters are then sorted dependent on their centroid value and coloured accordingly to a predefined heatmap colour palette. To finally score each joint, the pixels in the segmented joint area and their assigned cluster are summed and normalized by the area's amount of pixels and $\mathrm{k}$.

Results: 271 of the patients were investigated by the newly developed method and compared with the CE scoring. 6426 joints were labeled as healthy whereas 1162 joints were either labeled as swollen, tender or both. The result over all investigated patients for $\mathrm{k}=9$ is summed in table 1 . It is observable that every average and median healthy value is lower than the corresponding affected value.
Table 1. Resulting scores for $k=9$ for all 271 patients.

\begin{tabular}{lcccccc}
\hline Feature Statistic value & Amplitude & \multicolumn{3}{c}{ Mean } & \multicolumn{3}{c}{ Slope } \\
\hline & Healthy & Affected & Healthy & Affected & Healthy & Affected \\
\hline Average & 0.503 & 0.528 & 0.486 & 0.509 & 0.395 & 0.414 \\
Median & 0.496 & 0.532 & 0.482 & 0.505 & 0.389 & 0.415 \\
\hline
\end{tabular}

Conclusion: FOI is an innovative method that detects early changes in vascularization of the hands. So, this method can be useful in early detection of arthritis especially in risk populations such as PsO patients. The results of the objective scoring method show that a clear distinction between healthy and affected joints is possible with the average scores as well as the median values. However, if the range of the scores is considered, the overlap between healthy and affected is not neglectable. Thus, the current scoring system can be used as an indicator but not as a single classification marker. Nevertheless, the research at hand has shown the expected outcome and motivates further development on the heatmap approach. Disclosure of Interests: Lukas Zerweck: None declared, Ulf Henkemeier: None declared, Phuong-Ha Nguyen: None declared, Tanja Rossmanith Grant/research support from: Janssen, BMS, LEO, Pfizer, Andreas Pippow: None declared, Harald Burkhardt Grant/research support from: Pfizer, Roche, Abbvie, Consultant of: Sanofi, Pfizer, Roche, Abbvie, Boehringer Ingelheim, UCB, Eli Lilly, Chugai, Bristol Myer Scripps, Janssen, and Novartis, Speakers bureau: Sanofi, Pfizer, Roche, Abbvie, Boehringer Ingelheim, UCB, Eli Lilly, Chugai, Bristol Myer Scripps, Janssen, and Novartis, Frank Behrens Grant/research support from: Pfizer, Janssen, Chugai, Celgene, Lilly and Roche, Consultant of: Pfizer, AbbVie, Sanofi, Lilly, Novartis, Genzyme, Boehringer, Janssen, MSD, Celgene, Roche and Chugai, Michaela Köhm Grant/research support from: Pfizer, Janssen, BMS, LEO, Consultant of: BMS, Pfizer, Speakers bureau: Pfizer, BMS, Janssen, Novartis DOI: 10.1136/annrheumdis-2020-eular.5371

\section{AB1139 DIAGNOSTICIS AND PROGNOSTICIS SIGNIFICANCE OF CHEST CT EVALUATION OF SMALL PULMONARY VESSELS IN CONNECTIVE TISSUE DISEASES WITH PULMONARY ARTERIAL HYPERTENSION.}

Y. Zhang ${ }^{1}$, N. Zhang ${ }^{1}$, Y. Zhu ${ }^{2}$, Q. Wang ${ }^{2}$, L. Zhou ${ }^{2} .{ }^{1}$ Nanjing Medical University, Nanjing, China; ${ }^{2}$ The First Affiliated Hospital of Nanjing Medical University, Nanjing, China

Background: Pulmonary arterial hypertension (PAH) is a fatal complication of connective tissue diseases (CTDs). Chest CT has been increasingly used in the evaluation of patients with suspected $\mathrm{PH}$ noninvasively but there is a paucity of studies.

Objectives: Our study was aimed to investigate the cross-sectional area (CSA) of small pulmonary vessels on chest $\mathrm{CT}$ for the diagnosis and prognosis of CTD-PAH.

Methods: This retrospective study analyzed the data of thirty-four patients with CTD-PAH who were diagnosed by right heart catheterization $(\mathrm{RHC})$ and underwent chest CT between March 2011 and October 2019. We measured the percentage of total CSA of vessels $<5 \mathrm{~mm}^{2}$ and $5-10 \mathrm{~mm}^{2}$ as a percentage of total lung area $\left(\% \mathrm{CSA}_{<5}\right.$ and $\left.\% \mathrm{CSA}_{5-10}\right)$ on Chest $\mathrm{CT}$. Furthermore, the association of $\%$ CSA with mean pulmonary artery pressure (mPAP) was also investigated. Besides, these patients were followed up until October 2019, and Kaplan-Meier survival curves were generated for the evaluation of prognosis.

Results: Patients with CTD-PAH had significantly higher $\% \mathrm{CSA}_{5-10}$ than CTD$\mathrm{nPAH}(\mathrm{p}=0.001), \% \mathrm{CSA}_{5-10}$ in CTD-S-PAH and IPAH was significantly higher than CTD-LM-PAH and COPD-PH $(p<0.01)$. There was a positive correlation between $\%_{\mathrm{CSA}_{5-10}}$ and mPAP in CTD-PAH $(r=0.447, p=0.008)$. Considering $\% \mathrm{CSA}_{5-10}$ above 0.38 as a threshold level, the sensitivity and specificity were found to be 0.824 and 0.706 , respectively. Patients with $\% \mathrm{CSA}_{5-10} \geq 0.38$ had a lower survival rate than those with $\% \mathrm{CSA}_{5-10}<0.38(\mathrm{p}=0.049)$.

Conclusion: Quantitative parameter, $\% \mathrm{CSA}_{5-10}$ on Chest CT might serve a crucial differential diagnostic tool for different types of $\mathrm{PH}$. \%CSA ${ }_{5-10} \geq 0.38$ is a prognostic indicator for evaluation of CTD-PAH.

\section{References:}

[1] Galie N, Humbert M, Vachiery JL, et al. 2015 ESC/ERS Guidelines for the Diagnosis and Treatment of Pulmonary Hypertension. Rev Esp Cardiol (Engl Ed). 2016;69(2):177.

[2] Siddiqui I, Rajagopal S, Brucker A, et al. Clinical and Echocardiographic Predictors of Outcomes in Patients With Pulmonary Hypertension. Am J Cardiol. 2018;122(5):872-878.

[3] Coste F, Dournes G, Dromer C, et al. CT evaluation of small pulmonary vessels area in patients with COPD with severe pulmonary hypertension. Thorax. 2016;71(9):830-837. 